\title{
Patterns of a slow air-water flow in a semispherical container
}

Balci, Adnan; Brøns, Morten; Herrada, Miguel A.; Shtern, Vladimir N.

Published in:

European Journal of Mechanics B - Fluids

Link to article, DOI:

10.1016/j.euromechflu.2016.03.004

Publication date:

2016

Document Version

Peer reviewed version

Link back to DTU Orbit

Citation (APA):

Balci, A., Brøns, M., Herrada, M. A., \& Shtern, V. N. (2016). Patterns of a slow air-water flow in a semispherical container. European Journal of Mechanics B - Fluids, 58, 1-8. https://doi.org/10.1016/j.euromechflu.2016.03.004

\section{General rights}

Copyright and moral rights for the publications made accessible in the public portal are retained by the authors and/or other copyright owners and it is a condition of accessing publications that users recognise and abide by the legal requirements associated with these rights.

- Users may download and print one copy of any publication from the public portal for the purpose of private study or research.

- You may not further distribute the material or use it for any profit-making activity or commercial gain

- You may freely distribute the URL identifying the publication in the public portal 


\title{
Patterns of a slow air-water flow in a semispherical container
}

\author{
Adnan Balci ${ }^{1}$, Morten Brøns ${ }^{1}$, Miguel A. Herrada ${ }^{2}$ and Vladimir N. Shtern ${ }^{3}$ \\ ${ }^{1}$ DTU Compute, Technical University of Denmark 2800 Kgs. Lyngby, Denmark \\ ${ }^{2}$ E.S.I, Universidad de Sevilla, Camino de los Descubrimientos s/n 41092, Spain \\ ${ }^{3}$ Shtern Research and Consulting, Houston, Texas 77096, USA
}

This numerical study analyzes the development of eddies in a slow steady axisymmetric airwater flow in a sealed semispherical container, driven by a rotating top disk. As the water height, $H_{\mathrm{w}}$, increases, new flow cells emerge in both water and air. First, an eddy emerges near the axis-bottom intersection. Then this eddy expands and reaches the interface, inducing a new cell in the air flow. This cell appears as a thin near-axis layer which then expands and occupies the entire air domain. As the disk rotation intensifies at $H_{\mathrm{w}}=0.8$, the new air cell shrinks to the axis and disappears. The bulk water circulation becomes separated from the interface by a thin layer of water counter-circulation. These changes in the flow topology occur due to (a) competing effects of the air meridional flow and swirl, which drive meridional motions of opposite directions in water, and (b) feedback of water flow on the air flow. In contrast to flows in cylindrical and conical containers, there is no interaction with Moffatt corner vortices here.

Key words: Swirling motions, two-fluid flows, viscous incompressible fluids, sealed container, changes in flow topology 


\section{Introduction}

Moffatt [1] found a counter-intuitive phenomenon: a creeping flow can have a complex topology. An unbounded number of eddies can exist between two inclined plane. The strength and dimension of eddies decrease toward the edge. Eddies disappear as the angle between the planes enlarges and passes a threshold. Similar cellular flow patterns have been found in in a plane cavity [1, 2], cone [3], cylinder [4, 5], between concentric cones [6] and coaxial cylinders [7].

The mechanism of the Moffatt eddies is based on the dead-end geometry of a cavity and the dissipation of kinetic energy. The dead end induces a counterflow and the dissipation is smaller in a set of eddies than that in a global counterflow [8]. A different mechanism of eddy formation occurs in two-fluid swirling flows. In particular, it works in the cylindrical [9] and conical [10] sealed vertical containers where an air-water flow is driven by the rotating lid.

These flows recently attracted the attention of researchers due to possible applications in bioreactors. Aerial bioreactors are a novel technology for the growth of a tissue culture $[11,12]$. The rotating top disk induces a swirling motion of air. The air flow converges toward the reactor axis near the interface and drives the meridional circulation and slow rotation of water. The air circulation delivers oxygen, required for the tissue culture growth, to the interface. The water circulation enhances mixing of the dissolved oxygen with other ingredients. The tissue fraction is small compared to that of water and is neglected in the studies of flow pattern [12, 13]. The tissue growth is a time-consuming process requiring a slow motion of ingredients and small shear stresses. The air driving satisfies these requirements.

The mechanism of the eddy emergence in air-water flows is kinematic and based on the capability of swirl to generate the circulatory meridional motion. The rotating lid induces a 
centrifugal force which pushes the air to the periphery near the lid thus driving the clockwise meridional circulation of air which converges to the axis near the interface. The water meridional motion is driven by two factors, both provided by the air flow: (a) swirl and (b) meridional velocities at the interface.

Factors (a) and (b) tend to move water in opposite directions: the swirl (meridional) velocity drives the clockwise (anticlockwise) circulation. The swirl rapidly decays downward in a creeping flow [5]. Therefore if the water volume fraction, $\mathrm{H}_{\mathrm{w}}$, is small, factor (b) dominates and the bulk circulation of water is anticlockwise. As $\mathrm{H}_{w}$ increases, factor (a) becomes stronger and first induces a cell of the clockwise circulation near the bottom center. Then this cell expands and touches the interface. The velocity at the interface becomes reversed near the axis and drives a thin layer of anticlockwise circulation in the water.

In the cylindrical and conical containers, there are sets of the Moffatt eddies near the sidewall-bottom intersection. These eddies interact with those induced by the kinematic mechanism that results in multiple topological transformations of the flow as $\mathrm{H}_{\mathrm{w}}$ varies - up to eighteen changes of flow topology in a conical container [10].

A motivation of the current study is to exclude Moffatt eddies and thus to investigate the flow transformations occurring due to the kinematic mechanism only. To this end, the semispherical geometry of the container is relevant, having no corner where the Moffatt eddies could occur. In addition, the corner eddies elevate shear stresses thus deteriorating the tissue safety. This concern was our motivation to address the semispherical container whose smooth spherical wall helps reduce shear stresses. The semispherical container has not been yet addressed previously. 
In this paper we show that as the water-height-to-radius ratio $H_{\mathrm{w}}$ increases, the clockwise cell emerges near the axis-bottom intersection at $H_{\mathrm{w}}=0.666$. Then the water counter-circulation expands, reaches the interface and induces a counter-circulation in a thin near-axis layer of air. Next the clockwise circulation occupies the entire water domain and finally the anticlockwise circulation occupies the entire air domain as $H_{\mathrm{w}}$ approaches 1 .

In addition to the creeping flow, we consider the impact of moderately fast disk rotation, characterized by the Reynolds number $R e$. As $R e$ increases at $H_{\mathrm{w}}=0.8$, the anticlockwise circulation of air shrinks to the axis and disappears. A thin layer of water anticlockwise circulation develops separating the clockwise circulations of air and water. Physical reasons are provided for these topological transformations of the air-water flow.

In the rest of this paper, we formulate the problem (§2), describe the numerical technique (§3), explore the topological transformations of the creeping flow as $H_{\mathrm{w}}$ increases (§4), and how the flow pattern changes as $R e$ increases at $H_{\mathrm{w}}=0.8$ (§5), summarize the results in $\S 6$, and verify their grid-independence in the Appendix.

\section{Problem formulation}

1. Flow geometry.

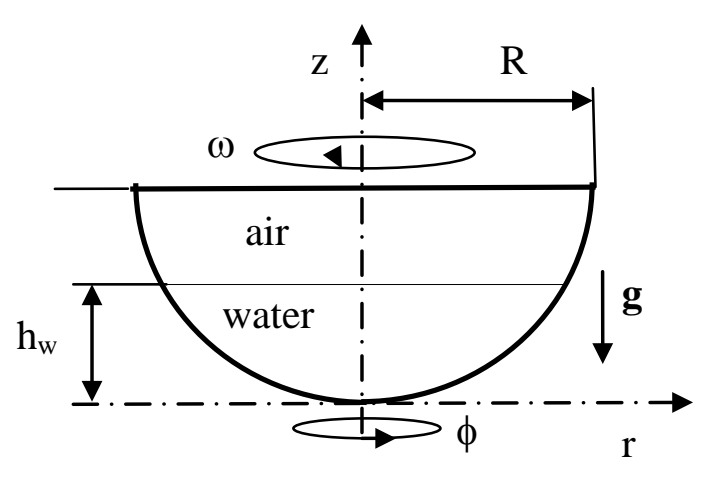


Fig. 1. Schematic of the problem. The lid only rotates.

Figure 1 is a problem schematic. The lower part, $0<\mathrm{z}<\mathrm{h}_{\mathrm{w}}$, of the semispherical container is filled with water, the upper part, $\mathrm{h}_{\mathrm{w}}<\mathrm{z}<\mathrm{R}$, is filled with air; $\mathrm{r}, \phi$ and $\mathrm{z}$ are cylindrical coordinates and $\mathbf{g}$ is the gravitational acceleration. The interface is depicted by the thin horizontal line, $\mathrm{z}=\mathrm{h}_{\mathrm{w}}$. The semispherical wall is stationary. The disk lid, located at $\mathrm{z}=\mathrm{R}$, rotates with angular velocity $\omega$; $\mathrm{R}$ is the disk and hemisphere radius, which serves as a length scale. The dimensionless control parameters are the water fraction, characterized by the water height $H_{\mathrm{w}}=\mathrm{h}_{\mathrm{w}} / \mathrm{R}$, and the Reynolds number, $R e=\omega \mathrm{R}^{2} / \mathrm{v}_{\mathrm{w}}$, characterizing the rotation strength; $v_{\mathrm{w}}$ is the kinematic viscosity of water. The motion is creeping if $R e<<1$.

One our goal is to explore the eddy development in the creping flow as $H_{\mathrm{w}}$ increases. The second goal is to explore the flow topological transformations as $R e$ increases at a fixed $H_{\mathrm{w}}$. To this end, $H_{\mathrm{w}}=0.8$ is chosen where the creeping flow is multicellular.

\subsection{Governing equations}

Using $R, \omega R$, and $\rho_{w} \omega^{2} R^{2}$ as scales for length, velocity, and pressure, respectively, renders all variables dimensionless. We consider steady axisymmetric flows of air and water both governed by the Navier-Stokes equations for a viscous incompressible fluid in the form used by Herrada \& Shtern [9],

$$
\begin{aligned}
& \mathrm{r}^{-1} \partial(\mathrm{ru}) / \partial \mathrm{r}+\partial \mathrm{w} / \partial \mathrm{z}=0, \\
& \mathrm{u} \partial \mathrm{u} / \partial \mathrm{r}+\mathrm{w} \partial \mathrm{u} / \partial \mathrm{z}=\mathrm{v}^{2} / \mathrm{r}-\rho_{\mathrm{n}} \partial \mathrm{p} / \partial \mathrm{r}+\mathrm{v}_{\mathrm{n}} \operatorname{Re}^{-1}\left(\nabla^{2} \mathrm{u}-\mathrm{u} / \mathrm{r}^{2}\right), \\
& \partial \mathrm{v} / \partial \mathrm{r}+\mathrm{w} \partial \mathrm{v} / \partial \mathrm{z}+\mathrm{uv} / \mathrm{r}=\mathrm{v}_{\mathrm{n}} \operatorname{Re}^{-1}\left(\nabla^{2} \mathrm{v}-\mathrm{v} / \mathrm{r}^{2}\right), \\
& \mathrm{u} \partial \mathrm{w} / \partial \mathrm{r}+\mathrm{w} \partial \mathrm{w} / \partial \mathrm{z}=-\rho_{\mathrm{n}} \partial \mathrm{p} / \partial \mathrm{z}+\mathrm{v}_{\mathrm{n}} \operatorname{Re}^{-1}\left(\nabla^{2} \mathrm{w}\right),
\end{aligned}
$$


where $\nabla^{2} \equiv \mathrm{r}^{-1} \partial(\mathrm{r} \partial / \partial \mathrm{r}) / \partial \mathrm{r}+\partial^{2} / \partial \mathrm{z}^{2}$, (u, v, w) are the radial, azimuthal and axial velocity components respectively, and $\mathrm{p}$ is pressure reduced by the hydrostatic contribution. The coefficients, $\rho_{\mathrm{n}}$ and $v_{\mathrm{n}}$, both equal to 1 for $\mathrm{n}=1$ (in water) while $\rho_{\mathrm{n}}=\rho_{\mathrm{w}} / \rho_{\mathrm{a}}$ and $v_{\mathrm{n}}=v_{\mathrm{a}} / v_{\mathrm{w}}$ for $\mathrm{n}=$ 2 (in air); $v_{\mathrm{w}}=10^{-6} \mathrm{~m}^{2} / \mathrm{s}$ and $v_{\mathrm{a}}=15 \times 10^{-6} \mathrm{~m}^{2} / \mathrm{s}$ are the kinematic viscosities; $\rho_{\mathrm{w}}=1000 \mathrm{~kg} / \mathrm{m}^{3}$ and $\rho_{\mathrm{a}}=1.22 \mathrm{~kg} / \mathrm{m}^{3}$ are the densities; subscripts "a" and " $\mathrm{w}$ " are abbreviations for "air" and "water". The air and water properties correspond to atmospheric pressure and room temperature.

In a creeping flow the interface is undisturbed. Herrada and Shtern [9] found that the interface deformation also is negligibly small for moderate Re typical of bioreactor flows. No significant deformation of the interface was observed in the experiment of Lo Jacomo et al [13]. The physical reason is the small air-to-water density ratio. Based on this, the interface is considered undisturbed in this study: $\mathrm{z}=\mathrm{z}_{\mathrm{i}}=H_{\mathrm{w}}$.

\subsection{Boundary conditions}

Equations (1)-(4) are solved under the following boundary conditions:

(i) Regularity at the axis, $0<\mathrm{z}<1, \mathrm{r}=0$ : $\mathrm{u}=\mathrm{v}=0, \partial \mathrm{w} / \partial \mathrm{r}=0$.

(ii) No-slip conditions, $\mathrm{u}=\mathrm{w}=0$ and $\mathrm{v}=\mathrm{r}$, at the rotating disk, $0<\mathrm{r}<1, \mathrm{z}=1$.

(iii) No-slip conditions, $\mathrm{u}=\mathrm{v}=\mathrm{w}=0$, at the spherical wall, $0<\mathrm{r}<1, \mathrm{z}=1-\left(1-\mathrm{r}^{2}\right)^{1 / 2}$.

(iv) Continuity of all the velocity and stress components at the air-water interface. Since the interface is a streamline, the normal-to-interface velocity is zero: $\mathrm{w}=0$ at $z=H_{\mathrm{w}}$.

\subsection{Reduced problem}

As $R e \rightarrow 0$, the nonlinear terms become negligibly small compared with the linear terms and can be omitted in equation (3), which reduces to

$$
\nabla^{2} \mathrm{v}-\mathrm{v} / \mathrm{r}^{2}=0
$$


This equation has a nonzero solution due to the boundary condition at the disk: $\mathrm{v}=\mathrm{r}$ at $\mathrm{z}=1$. At the spherical wall, the swirl velocity is zero, $v=0$. At the interface, $v$ and the swirl shear stresses are continuous: $\mathrm{v}_{\mathrm{w}}=\mathrm{v}_{\mathrm{a}}$ and $\partial \mathrm{v}_{\mathrm{w}} / \partial \mathrm{z}=\mu_{\mathrm{r}} \partial \mathrm{v}_{\mathrm{a}} / \partial \mathrm{z}$ at $\mathrm{z}=H_{\mathrm{w}} ; \mu_{\mathrm{r}}=v_{\mathrm{n}} / \rho_{\mathrm{n}}$. Thus the problem for the swirl velocity becomes separated from the problem for the meridional motion in the limiting case as $R e \rightarrow 0$, similar to that in the one-fluid problem studied by Hills [5]. We first solve this linear problem for swirl and then address the problem for the meridional motion.

Since the boundary conditions are uniform for the meridional motion, it is only driven by the centrifugal force corresponding to term $\mathrm{v}^{2} / \mathrm{r}$ in equation (2). This term must be preserved, while the other nonlinear terms can be omitted in the limiting case as $R e \rightarrow 0$. Then introducing $\mathrm{u}^{*}=$ $\mathrm{u} / \operatorname{Re}$ and $\mathrm{w}^{*}=\mathrm{w} / \operatorname{Re}$ reduces equations (1), (2), and (4) to

$$
\begin{aligned}
& \mathrm{r}^{-1} \partial\left(\mathrm{ru}^{*}\right) / \partial \mathrm{r}+\partial \mathrm{w}^{*} / \partial \mathrm{z}=0, \\
& \rho_{\mathrm{n}} \partial \mathrm{p} / \partial \mathrm{r}-\mathrm{v}^{2} / \mathrm{r}=\mathrm{v}_{\mathrm{n}}\left(\nabla^{2} \mathrm{u}^{*}-\mathrm{u}^{*} / \mathrm{r}^{2}\right) \\
& \rho_{\mathrm{n}} \partial \mathrm{p} / \partial \mathrm{z}=v_{\mathrm{n}} \nabla^{2} \mathrm{w}^{*}
\end{aligned}
$$

It is interesting that the entire problem formally is nonlinear despite the motion is creeping, but can be reduced to the two linear problems: one for the swirl velocity (5) and the other for the meridional motion (6)-(8). After solving problem (5), the "source" term, $v^{2} / r$ in equation (7), is prescribed, so the problem for the meridional motion also is linear. This feature is also similar to that in the flow studied by Hills [5].

\section{Numerical procedure}

\subsection{Transformation of equations}

The number of variables involved in the problem is reduced by introducing a stream-

function-vorticity-circulation form. System (1)-(4) is transformed into three equations for the 
Stokes stream function $\Psi, \mathrm{u}=-\mathrm{r}^{-1} \partial \Psi / \partial \mathrm{z}, \mathrm{w}=\mathrm{r}^{-1} \partial \Psi / \partial \mathrm{r}$, the azimuthal vorticity component, $\eta=$ $\partial \mathrm{u} / \partial \mathrm{z}-\partial \mathrm{w} / \partial \mathrm{r}$, and circulation, $\Gamma=\mathrm{rv}$ :

$$
\begin{gathered}
\nabla^{2} \Psi-2 \mathrm{r}^{-1} \partial \Psi / \partial \mathrm{r}=-\mathrm{r} \eta, \\
\mathrm{u} \partial \eta / \partial \mathrm{r}+\mathrm{w} \partial \eta / \partial \mathrm{z}-\mathrm{u} \eta / \mathrm{r}=2 \mathrm{r}^{-3} \Gamma \partial \Gamma / \partial \mathrm{z}+v_{\mathrm{n}} \operatorname{Re}^{-1}\left(\nabla^{2} \eta-\eta / \mathrm{r}^{2}\right), \\
\mathrm{u} \partial \Gamma / \partial \mathrm{r}+\mathrm{w} \partial \Gamma / \partial \mathrm{z}=v_{\mathrm{n}} \operatorname{Re}^{-1}\left(\nabla^{2} \Gamma-2 \mathrm{r}^{-1} \partial \Gamma / \partial \mathrm{r}\right),
\end{gathered}
$$

These equations are solved applying the boundary conditions for $\Psi, \eta$ and $\Gamma$ which follows from those listed in §2.3. In particular, $\Psi=0$ at the container walls, axis and interface while $\Gamma=$ 0 at the semispherical wall and axis, $\Gamma=\mathrm{r}^{2}$ at the lid and $\Gamma$ is continuous at the interface.

\section{Linear problem}

In the limiting case as $R e \rightarrow 0$, introducing $\eta^{*}=\eta / R e$ and $\Psi^{*}=\Psi / \operatorname{Re}$ reduces equations (9)(11) to

$$
\begin{aligned}
& \nabla^{2} \Psi^{*}-2 \mathrm{r}^{-1} \partial \Psi * / \partial \mathrm{r}=-\mathrm{r} \eta^{*}, \\
& 2 \mathrm{r}^{-3} \Gamma \partial \Gamma / \partial \mathrm{z}+v_{\mathrm{n}}\left(\nabla^{2} \eta^{*}-\eta^{*} / \mathrm{r}^{2}\right)=0, \\
& \nabla^{2} \Gamma-2 \mathrm{r}^{-1} \partial \Gamma / \partial \mathrm{r}=0 .
\end{aligned}
$$

\subsection{Discretization}

A boundary-fitted coordinate system is used to calculate the problem. Both the water and air regions are mapped onto the fixed rectangular domains (a) $0 \leq \zeta \leq 1,0 \leq \xi_{\mathrm{w}} \leq H_{\mathrm{w}}$, and (b) $0 \leq \zeta$ $\leq 1, H_{\mathrm{w}} \leq \xi_{\mathrm{a}} \leq 1$. To this end, we perform the coordinate transformations $\xi_{\mathrm{w}, \mathrm{a}}=\mathrm{z}, \zeta=$ $\mathrm{r} / \sin (\operatorname{acos}(1-\mathrm{z}))$. The rectangular domains are discretized by using a set of $\mathrm{n}_{\xi \mathrm{w}}$ and $\mathrm{n}_{\xi \mathrm{a}}$ Chebychev spectral collocation points in the $\xi$ direction. The $\zeta$ interval is discretized using a set of $\mathrm{n}_{\zeta}$ Chebychev spectral collocation points. 


\subsection{Nonlinear problem}

The Newton iterative procedure is used to solve the discretized non-linear problem derived from (9)-(11) and their corresponding boundary conditions. Given an initial solution guess, a new approximate solution is found by solving the system of $3\left(n_{\xi w}+n_{\xi a}\right) \times n_{\varphi}$ linear algebraic equations for $\Psi, \eta$ and $\Gamma$ at the collocation points resulting from the Newton linearization. The simulation is started with a small value of the Reynolds number and the flow at rest. Once the approximate solution has converged, the converged solution is used as a new guess for a new run with a higher Reynolds number.

\subsection{Advantages of Chebyshev grid}

In the reduced problem, the discretized problem consists of (i) system of $\left(\mathrm{n}_{\xi \mathrm{w}}+\mathrm{n}_{\xi \mathrm{a}}\right) \times \mathrm{n}_{\varphi}$ linear algebraic equations for $\Gamma$ and (ii) a system of $2\left(\mathrm{n}_{\xi \mathrm{w}}+\mathrm{n}_{\xi \mathrm{a}}\right) \times \mathrm{n}_{\zeta}$ linear algebraic equations for $\Psi^{*}$ and $\eta^{*}$ which are solved sequentially. All linear systems, in the full and reduced problems, are solved directly applying the standard procedure of matrix inversion.

For the presented results, the simulations are done mostly with $n_{\zeta \mathrm{w}}=60, \mathrm{n}_{\xi \mathrm{a}}=60$, and $\mathrm{n}_{\zeta}=40$ (standard grid). In order to verify the grid independence, some runs have been carried out at larger $\mathrm{n}_{\xi \mathrm{w}}, \mathrm{n}_{\xi \mathrm{a}}$, and $\mathrm{n}_{\zeta}$. The comparison of the results obtained with the standard and fine grids can be found in the Appendix.

Since the Chebyshev grid points concentrate near the interface from both sides, the approach is adequate to resolve thin circulation layers, located near the interface, even using moderate numbers of grid points. The Chebyshev grid points concentrate near the spherical wall that helps resolve the emergence of new cell near the axis-bottom intersection in the water flow. The Chebyshev grid points also concentrate near the axis that helps resolve the anticlockwise circulation emerging near the axis-interface intersections in the air flow as $H_{\mathrm{w}}$ varies. 


\section{The development of new cells in a creeping flow}

\subsection{Development of clockwise circulation in the water flow}

Figure 2(a) depicts streamlines of the meridional motion, i.e., contours $\Psi=$ constant, at the water height $H_{\mathrm{w}}=0.66$. The stream function range is $\Psi_{\min }=-2.03 \times 10^{-8}<\Psi<2.33 \times 10^{-6}=\Psi_{\max }$ in Fig. 2(a). The $\Psi_{\min }\left(\Psi_{\max }\right)$ value is achieved at the center of the air (water) flow. The arrows indicate the flow directions. The rotating disk generates a centrifugal force, which pushes the air to the periphery near $\mathrm{z}=1$ and thus develops the clockwise circulation in region $\mathrm{CR}_{\mathrm{a} 1}$. Contours, corresponding to the clockwise (anticlockwise) circulation are depicted hereafter by the light (dark) curves which are blue online.

The air flow converges to axis near the interface, $\mathrm{z}=H_{\mathrm{w}}$, and drives the water anticlockwise circulation in region $\mathrm{CR}_{\mathrm{w} 1}$. This flow topology remains invariant as $H_{\mathrm{w}}$ decreases down to zero. The physical reason for the anticlockwise circulation is a weak rotation of water for small $H_{\mathrm{w}}$.
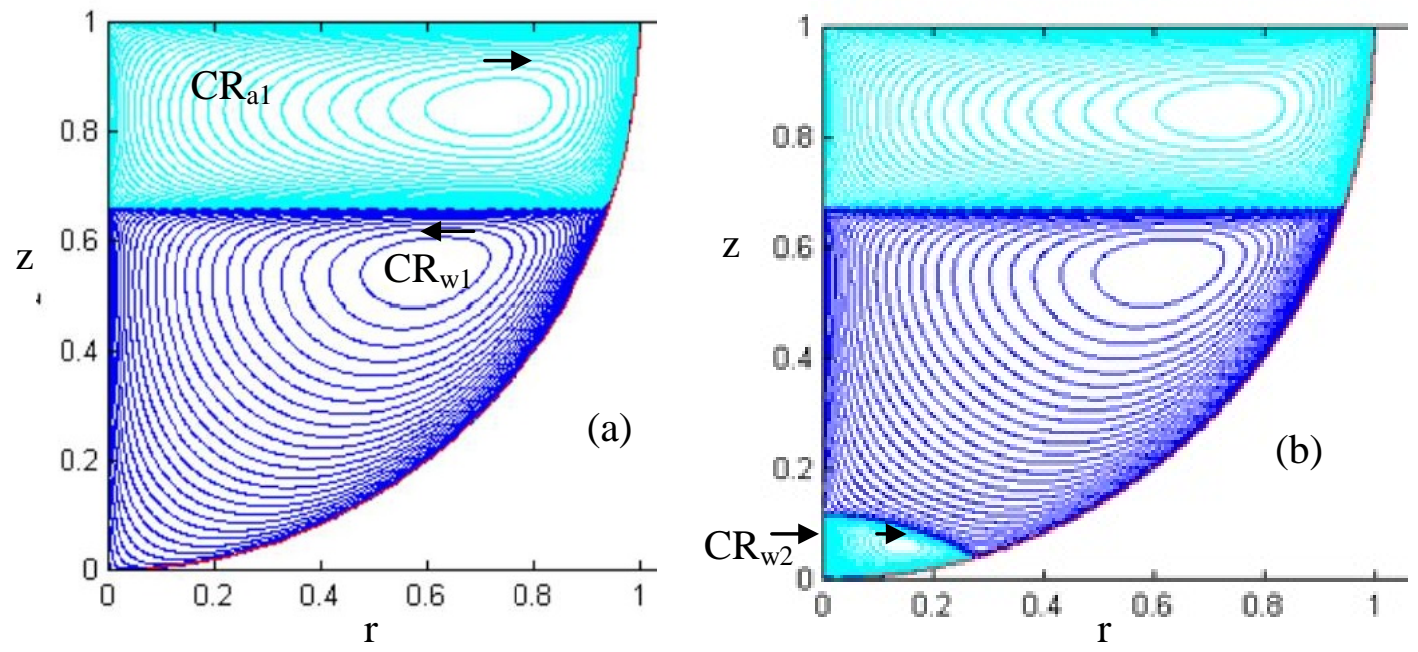

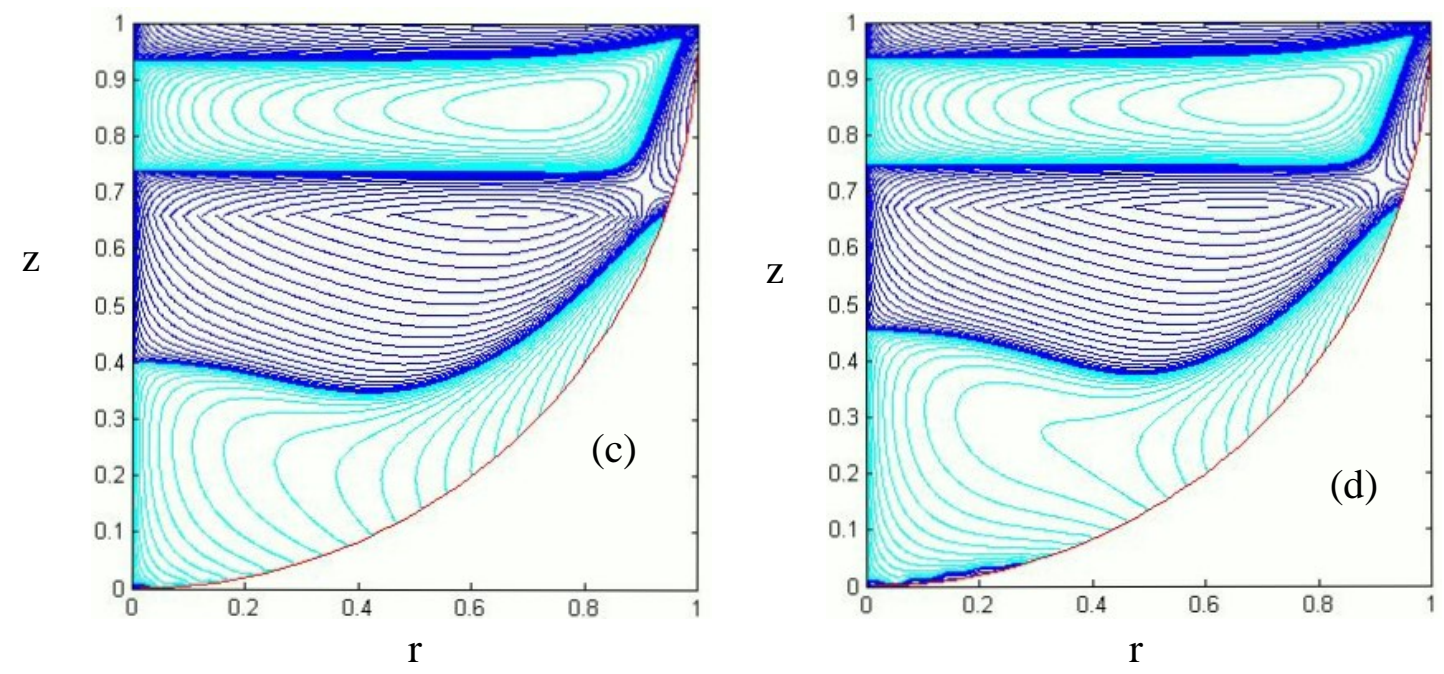

Fig. 2. Emergence of counter-circulation $\mathrm{CR}_{\mathrm{w} 2}$ in water as $H_{\mathrm{w}}$ increases from 0.66 (a, c) to 0.67 (b. d). Contours are shown of $\Psi=$ constant (a,b) and $\eta=$ constant (c,d).

For small $H_{\mathrm{w}}$, the centrifugal force contribution to the meridional motion of water is negligible compared with the air-meridional-flow impact because the swirl velocity at the interface rapidly decays as $H_{\mathrm{w}}$ decreases. Figure 3 illustrates this decay presenting the dependence of the maximal swirl velocity at the interface, $\mathrm{v}_{\mathrm{im}}$, on $H_{\mathrm{w}}$. Near $H_{\mathrm{w}}=0.6$, the decay rate is minimal, the dependence is nearly exponential and can be fitted by the relation, $\mathrm{v}_{\mathrm{im}}=$ $0.00452 \exp \left[5.05\left(H_{\mathrm{w}}-0.6\right)\right]$.

As $H_{\mathrm{w}}$ increases, the effect of swirl becomes significant and results in the emergence of clockwise circulation near the axis-bottom intersection in region $\mathrm{CR}_{\mathrm{w} 2}$ shown in Fig. 2(b) at $H_{\mathrm{w}}$ 0.67. An interpolation yields that this emergence occurs at $H_{\mathrm{w}}=H_{\mathrm{ww}}=0.666$. The interpolation was made using the distribution of velocity $\mathrm{w}$ at the axis. Figure 4 depicts $\mathrm{w}(\mathrm{z})$ at $\mathrm{r}$ $=0$ for a few values of $H_{\mathrm{w}}$ shown near the curves. We plot $\mathrm{w}^{1 / 3}$ in Fig. 4 in order to better observe small magnitudes of $\mathrm{w}$ typical of VB regions. In particular, the $\mathrm{w}>0$ range near $\mathrm{z}=0$ of the $H_{\mathrm{w}}=0.67$ curve in Fig. 4(a) corresponds to region $\mathrm{CR}_{\mathrm{w} 2}$ shown in Fig. 2(b). 


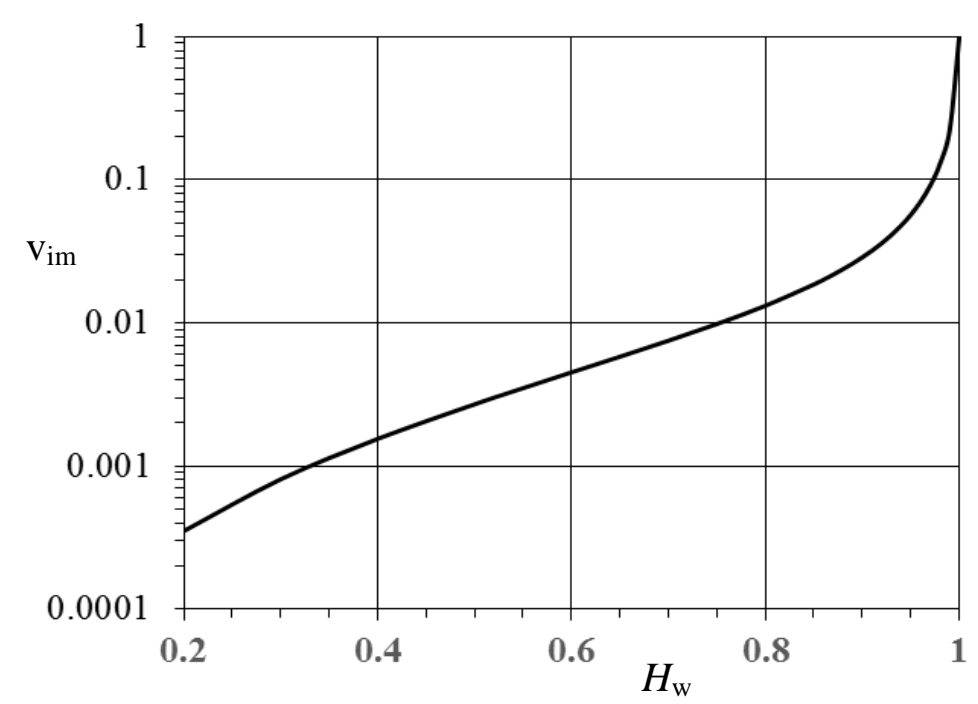

Fig. 3. Dependence on water height $H_{\mathrm{w}}$ of maximal swirl velocity at the interface $\mathrm{v}_{\mathrm{im}}$.
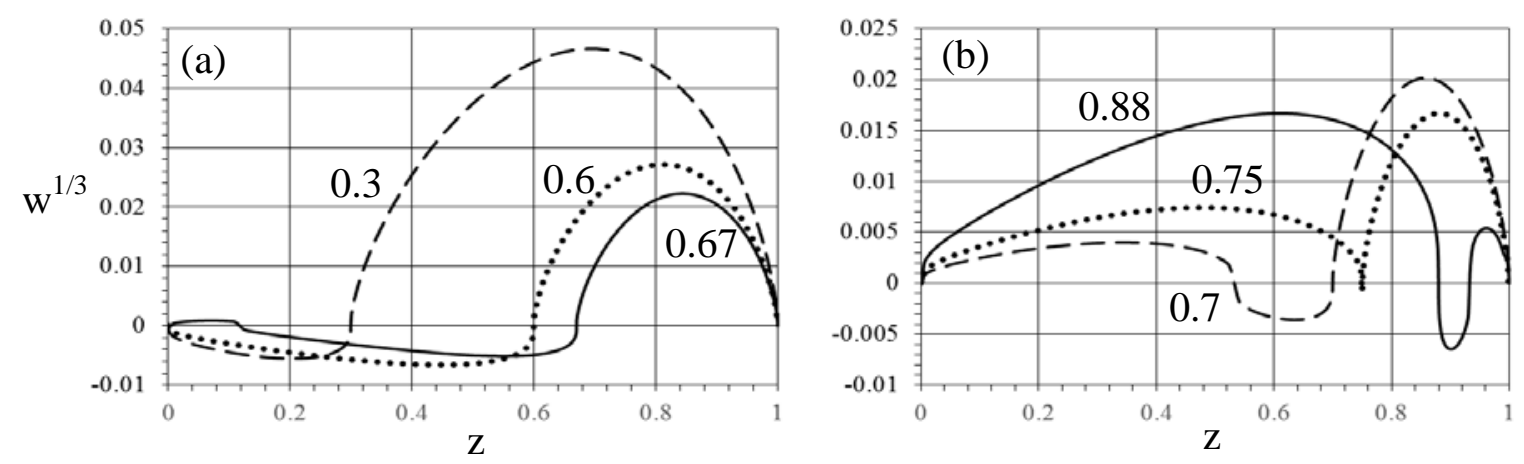

Fig. 4. Distribution of velocity w at the axis at $H_{\mathrm{w}}$ values shown near the curves.

This change in the flow topology is an example of the corner bubble-creation topological bifurcation studied by Brøns et al. [14, 15]. Its physical mechanism is the following. As Fig. 2(a) shows, the water flow in $\mathrm{CR}_{\mathrm{w} 1}$ is directed upward near the wall thus preventing the swirl penetration in the water depth. In contrast, the water flow is directed downward near the axis, transporting angular momentum to the bottom. Therefore, the swirl effect is the most prominent in the water flow near the axis. As the rotation intensifies, the cyclostrophic balance, $\partial \mathrm{p} / \partial \mathrm{r}=$ $\mathrm{v}^{2} / \mathrm{r}$, becomes approximately established. This balance, typical of a fast flow, can also occur in a creeping motion as Herrada \& Shtern [9] showed. 
Since the swirl velocity, $\mathrm{v}$, is zero at the wall due to the no-slip, the centrifugal force, $\mathrm{v}^{2} / \mathrm{r}$, diminishes near the bottom-axis intersection, and the radial gradient of pressure, $\partial \mathrm{p} / \partial \mathrm{r}$, becoming unbalanced by the centrifugal force, drives a secondary flow, converging to the axis near the wall, similar to that occurring in a high-speed swirling motion (Bödewadt [16]). This causes that region $\mathrm{CR}_{\mathrm{w} 2}$ develops where the water circulation is opposite to that in region $\mathrm{CR}_{\mathrm{w} 1}$ as Fig. 2(b) illustrates.

Figures 2(c) and 2(d) show contours of azimuthal vorticity, $\eta=$ constant, for the same $H_{\mathrm{w}}$ values for which streamlines are depicted in Figs. 2(a) and 2(b) respectively. Contours, corresponding to $\eta>0(\eta<0)$ are depicted by the light (dark) curves which are blue online. The vorticity is discontinuous at the interface and accordingly $\eta$-contours are disconnected at $\mathrm{z}=H_{\mathrm{w}}$. The emergence of region $\mathrm{CR}_{\mathrm{w} 2}$ in Fig. 2(b) corresponds to the emergence of the thin dark layer near the sidewall at small $\mathrm{z}$ in Fig. 2(d).

\subsection{Topological changes in the air flow}
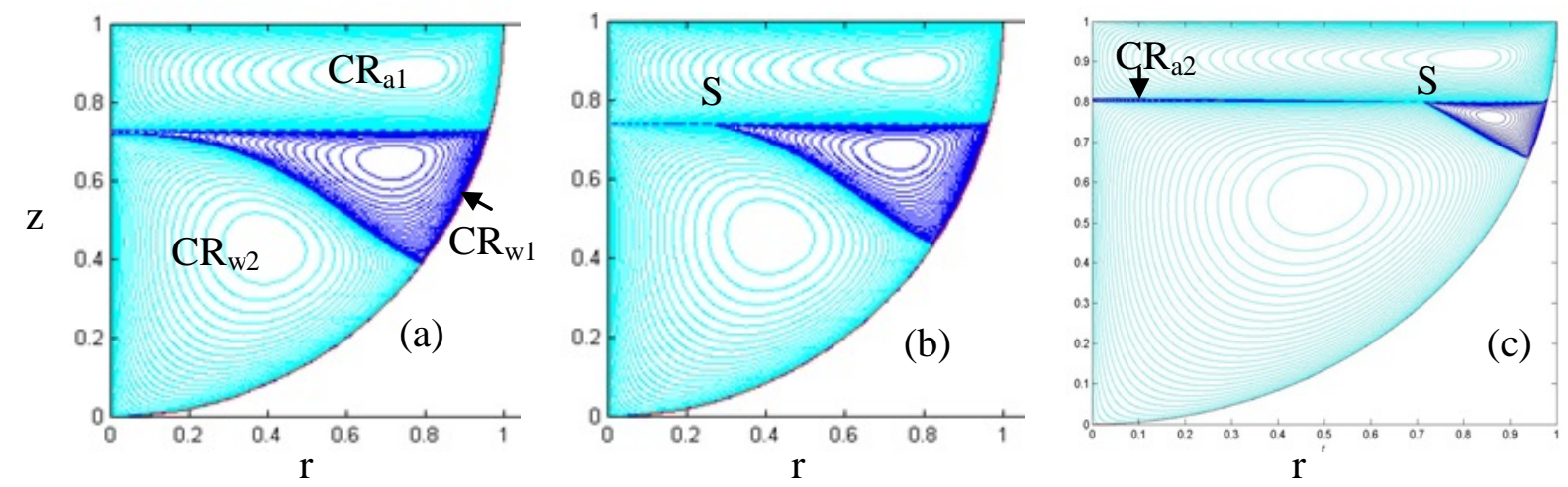

Fig. 5. Emergence of counter-circulation $\mathrm{CR}_{\mathrm{a} 2}$ in air as $H_{\mathrm{w}}$ increases; $H_{\mathrm{w}}=0.73$ (a), 0.74 (b) and 0.8 (c).

As $H_{\mathrm{w}}$ further increases, region $\mathrm{CR}_{\mathrm{w} 2}$ expands upward (Fig. 5a), reaches the interface-axis intersection point at $H_{\mathrm{w}}=H_{\mathrm{wa}}=0.732$ and then expands along the interface (Fig. 5b and 5c), reversing velocity u there. This change is known as a corner-crossing topological bifurcation 
[14]. The water flow near the interface-axis intersection, corresponding to the $\mathrm{u}>0$ range, drives the anticlockwise circulation of air in a thin circulation region, $\mathrm{CR}_{\mathrm{a} 2}$. Figure 5 depicts the corresponding transformations of streamline pattern.

Region $\mathrm{CR}_{\mathrm{w} 1}$ is topologically a bubble in Fig. 5(a) at $H_{\mathrm{w}}=0.73$, becomes a bubble-ring at $H_{\mathrm{w}}$ $=H_{\text {wa }}$, and then separates from the axis thus transforming in a ring as Figs. 5(b) at $H_{\mathrm{w}}=0.74$ and 5(c) at $H_{\mathrm{w}}=0.8$ depict. Region $\mathrm{CR}_{\mathrm{a} 2}$, where air circulates anticlockwise, is very thin, extends up to point S. $\mathrm{CR}_{\mathrm{a} 2}$ is hardly visible in Fig. 5(b) at $H_{\mathrm{w}}=0.74$ and clearly visible in Fig. 5 (c) at $H_{\mathrm{w}}=$ 0.8 .

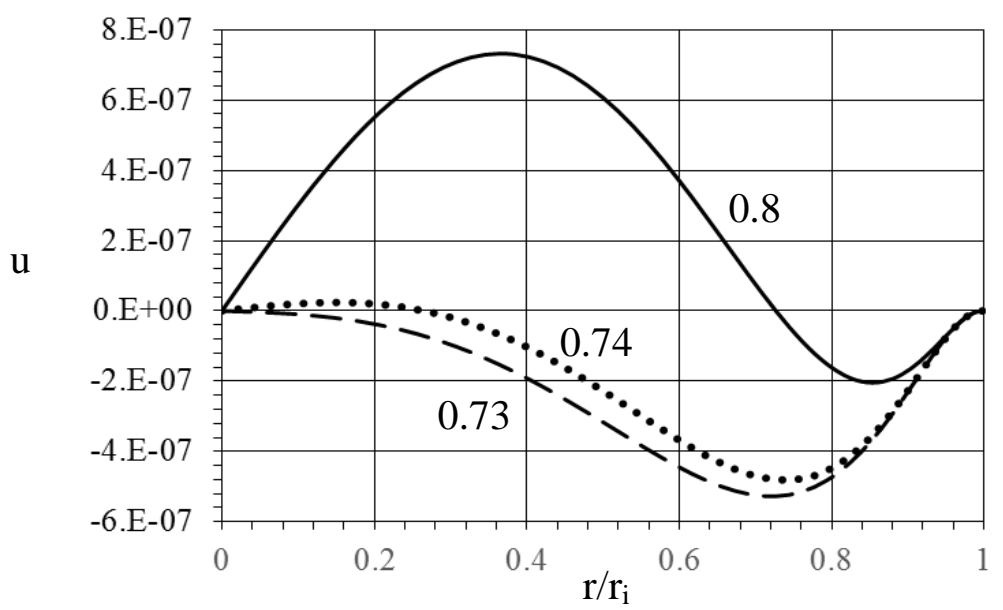

Fig. 6. Distribution of radial velocity u at the interface at $H_{\mathrm{w}}$ values shown near the curves.

Regions $\mathrm{CR}_{\mathrm{w} 1}, \mathrm{CR}_{\mathrm{w} 2}, \mathrm{CR}_{\mathrm{a} 1}$ and $\mathrm{CR}_{\mathrm{a} 2}$ all meet at the saddle point $\mathrm{S}$ which is a stagnation point of the meridional motion where $\mathrm{u}=\mathrm{w}=0$, but $\mathrm{v} \neq 0$. As $H_{\mathrm{w}}$ increases, region $\mathrm{CR}_{\mathrm{a} 2}$ rapidly expands in the radial direction as Fig. 5 illustrates. Figure 6 also demonstrates this feature by depicting the distribution of the radial velocity, $\mathrm{u}$, at the interface. Recall that $\mathrm{r}_{\mathrm{i}}$ is the radial extent of the interface which varies with $H_{\mathrm{w}}$. The $\mathrm{u}>0(\mathrm{u}<0)$ ranges of curves in Fig. 6 correspond to region $\mathrm{CR}_{\mathrm{a} 2}$ and $\mathrm{CR}_{\mathrm{w} 2}\left(\mathrm{CR}_{\mathrm{a} 1}\right.$ and $\left.\mathrm{CR}_{\mathrm{w} 1}\right)$. For $H_{\mathrm{w}}=0.73$ there is no part where $\mathrm{u}>$ 0. For $H_{\mathrm{w}}=0.74$ there is a short part with $\mathrm{u}>0$ for low values of $\mathrm{r} / \mathrm{r}_{\mathrm{i}}$ and for $H_{\mathrm{w}}=0.8$ has a 
large part with $\mathrm{u}>0$, in accordance with Fig. 5. The curve for $H_{\mathrm{w}}=0.75$ in Fig. 4(b) has a compact region where $\mathrm{w}<0$, which corresponds to $\mathrm{CR}_{\mathrm{a} 2}$ and shows that the $\mathrm{w}$ magnitude is very small, i.e., the meridional motion is nearly stagnant in $\mathrm{CR}_{\mathrm{a} 2}$ compared with that in the other circulation regions.

\subsection{Collapse of anticlockwise circulation in the water flow}

Figures 5 and 6 show that region $\mathrm{CR}_{\mathrm{w} 1}$ shrinks as $H_{\mathrm{w}}$ increases. Interpolation yields that region $\mathrm{CR}_{\mathrm{w} 1}$ totally disappears at $H_{\mathrm{w}}=H_{\mathrm{w} 3}=0.898$. This happens in a reverse corner-creation topological bifurcation as the point $\mathrm{S}$ approaches the wall. The interpolation was made using the distribution of radial velocity, $\mathrm{u}$, at the interface. Figure 7 depicts $\mathrm{u}(\mathrm{r})$ at $\mathrm{z}=H_{\mathrm{w}}$ for a few values of $H_{\mathrm{w}}$ shown near the curves. We plot $\mathrm{u}^{1 / 3}$ in Fig. 7 in order to better observe small magnitudes of u near the wall.

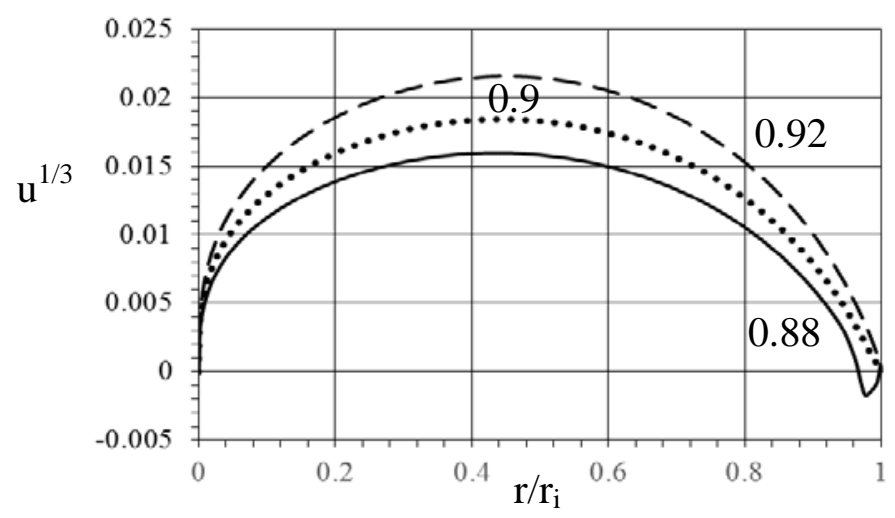

Fig. 7. Collapse of region $\mathrm{CR}_{\mathrm{w} 1}(\mathrm{u}<0)$ as $H_{\mathrm{w}}$, shown near the curves, increases. 

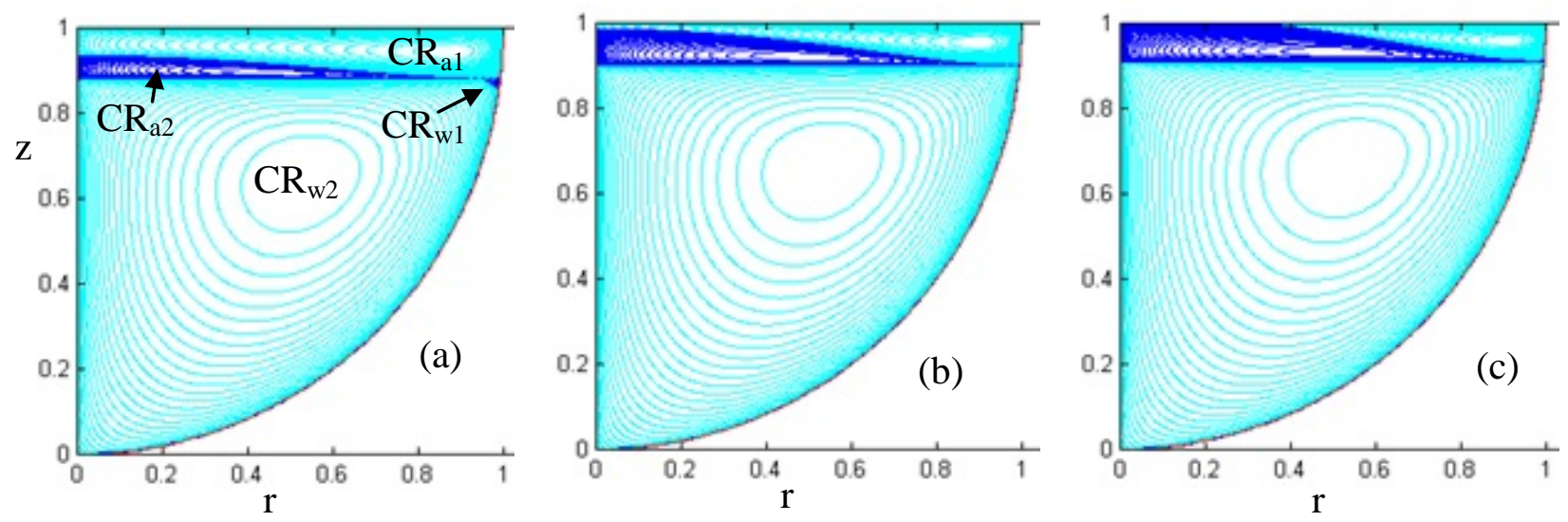

Fig. 8. Collapse of region $\mathrm{CR}_{\mathrm{w} 1}$ and separation of region $\mathrm{CR}_{\mathrm{a} 1}$ from the axis as $H_{\mathrm{w}}$ increases; $H_{\mathrm{w}}$ $=0.88(\mathrm{a}), 0.9$ (b) and 0.94 (c).

The $\mathrm{u}<0$ range near $\mathrm{r}=\mathrm{r}_{\mathrm{i}}$ at the $H_{\mathrm{w}}=0.88$ curve in Fig. 7 corresponds to region $\mathrm{CR}_{\mathrm{w} 1}$. Figure 8 depicts the streamline patterns (a) with and (b) without region $\mathrm{CR}_{\mathrm{w} 1}$. The water circulation is totally reversed in Figs. 8(b) and 8(c) compared with that in Fig. 2(a).

\subsection{Collapse of clockwise circulation in the air flow}

As $H_{\mathrm{w}}$ further increases, region $\mathrm{CR}_{\mathrm{a} 2}$ expands, reaches the axis-disk intersection point at $H_{\mathrm{w}}=$ $H_{\mathrm{w} 4}=0.935$ and expands along the rotating disk as Fig. 8(c) illustrates. The topological bifurcation at $H_{\mathrm{w} 4}$ is again a corner-crossing bifurcation. Region $\mathrm{CR}_{\mathrm{a} 1}$ is topologically a bubble for $H_{\mathrm{w}}<H_{\mathrm{w} 4}$, a bubble-ring at $H_{\mathrm{w}}=H_{\mathrm{w} 4}$ and a ring for $H_{\mathrm{w}}>H_{\mathrm{w} 4}$. As $H_{\mathrm{w}} \rightarrow 1$, region $\mathrm{CR}_{\mathrm{a} 1}$ shrinks to the disk-wall intersection.

\section{Flow transformations as the Reynolds number increases}

To explore in what range of the Reynolds number, $R e$, the flow topology remains the same as that for the creeping flow, we come back from the reduced equations (§2.4) to the full equations (§2.2 and §2.3) and use numerical techniques for the nonlinear problem (§3).

To characterize the air flow strength, we introduce the Reynolds number based on the air viscosity, $R e_{\mathrm{a}}=\omega \mathrm{R}^{2} / v_{\mathrm{a}}=R e / v_{\mathrm{n}}$, where $v_{\mathrm{n}}=15$. From the disk down to the interface, the swirl 
velocity significantly drops. Therefore, to characterize the strength of water flow, we introduce the Reynolds number, $R e_{\mathrm{w}}$, based on the maximal swirl velocity at the interface, $\mathrm{v}_{\mathrm{im}} ; R e_{\mathrm{w}}=$ $\mathrm{v}_{\text {im }} R e$.

\begin{tabular}{|c|c|c|}
\hline$R e$ & $R e_{\mathrm{a}}$ & $R e_{\mathrm{w}}$ \\
\hline 0.1 & 0.007 & 0.00132 \\
\hline 8000 & 533 & 86.1 \\
\hline 10000 & 667 & 107 \\
\hline 18000 & 1200 & 226 \\
\hline
\end{tabular}

Table 1. The values of the Reynolds numbers characterizing the air $R e_{\mathrm{a}}$ and water $R e_{\mathrm{w}}$ flows.

Figure 9 depicts how the streamline pattern changes as Re increases. In Fig. 9(b) where Re $=8000$, the flow topology is the same as that for the creeping motion depicted in Fig. 9(a) where $R e=0.1$. In contrast, the topology is different in Fig. 9(c) where $R e=10000$. Interpolation yields that the flow topology changes at $R e=8870$. This is again a corner-crossing bifurcation where the point $\mathrm{S}$ moves toward the axis and is transformed to a stagnation point on the axis in the water. The interpolation was made using the distribution of radial velocity, u, at the interface. Figure 10 depicts $\mathrm{u}(\mathrm{r})$ at $R e=8000$, where the $\mathrm{u}>0$ range corresponds to region $\mathrm{CR}_{\mathrm{a} 2}$ in Fig. 9(b), and at $R e=10000$, where the $\mathrm{u}>0$ range (i.e., region $\mathrm{CR}_{\mathrm{a} 2}$ ) does not exist in accordance with Fig. 9(c).

For $R e$ corresponding to Fig. 9, Table 1 provides values of $R e_{\mathrm{a}}$ and $R e_{\mathrm{w}}$. Since these values are moderate, we expect no flow instability. Increasing Re further to 18000 as shown in Fig. 9(d), we note that the region $\mathrm{CR}_{\mathrm{w} 1}$ takes an interesting form: the z-extent of $\mathrm{CR}_{\mathrm{w} 1}$ has a local minimum near $r=0.65$. 

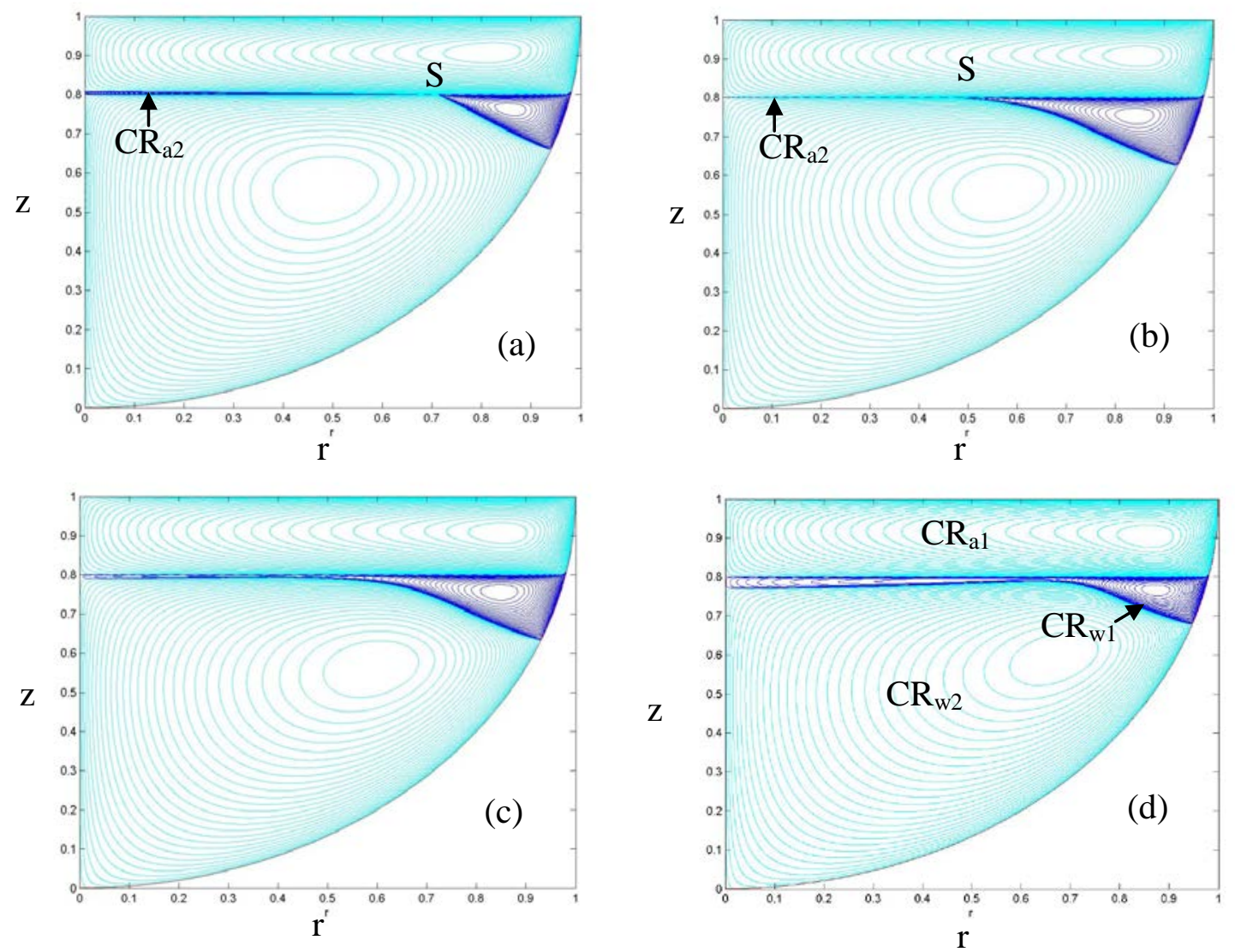

Fig. 9. Changes in the flow pattern as the rotation intensifies; $R e=0.1$ (a), 8000 (b), 10000 (c) and $18000(\mathrm{~d}) ; H_{\mathrm{w}}=0.8$.

To better understand the physical reason for the development of this 'neck' in region $\mathrm{CR}_{\mathrm{w} 1}$, we plot in Fig. 11 the distribution on the interface of the swirl, v, and radial, u, velocities, and the axial vorticity, $\omega_{\mathrm{z}}=\mathrm{r}^{-1} \partial(\mathrm{rv}) / \partial \mathrm{r}$. The vorticity and the radial velocity are scaled for convenient presentation in one figure. Curve 5u in Fig. 11 has a local maximum at the r value close to that for the 'neck' in Fig. 9(d). The peak of $\omega_{z}$ is also close to this location. We conclude that the effect of the water rotation is maximal in the $r$ range, corresponding to the 'neck'. The water clockwise circulation in region $\mathrm{CR}_{\mathrm{w} 2}$ tends to reduce the water anticlockwise circulation in region $\mathrm{CR}_{\mathrm{w} 1}$ thus developing the 'neck'. 
Our simulations indicate that the neck continues to exist and the water clockwise circulation does not reach the interface as $R e$ further increases, though the maximal z-extent of region $\mathrm{CR}_{\mathrm{w} 1}$ becomes smaller. This feature and the "thin circulation layer" shape of region $\mathrm{CR}_{\mathrm{w} 1}$ in Fig. 9(d) are similar to those observed in the cylindrical container [17].

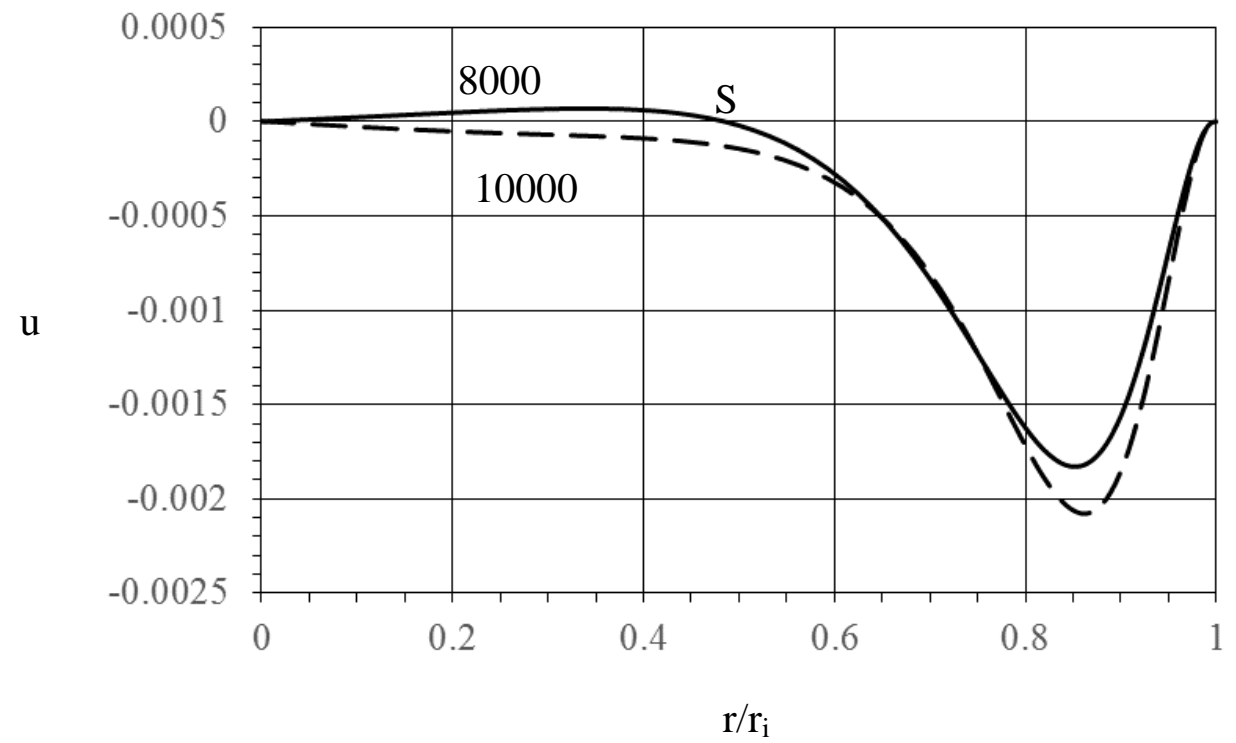

Fig. 10. Collapse of region $\mathrm{CR}_{\mathrm{a} 2}(\mathrm{u}>0)$ as $R e$ (shown near the curves) increases.

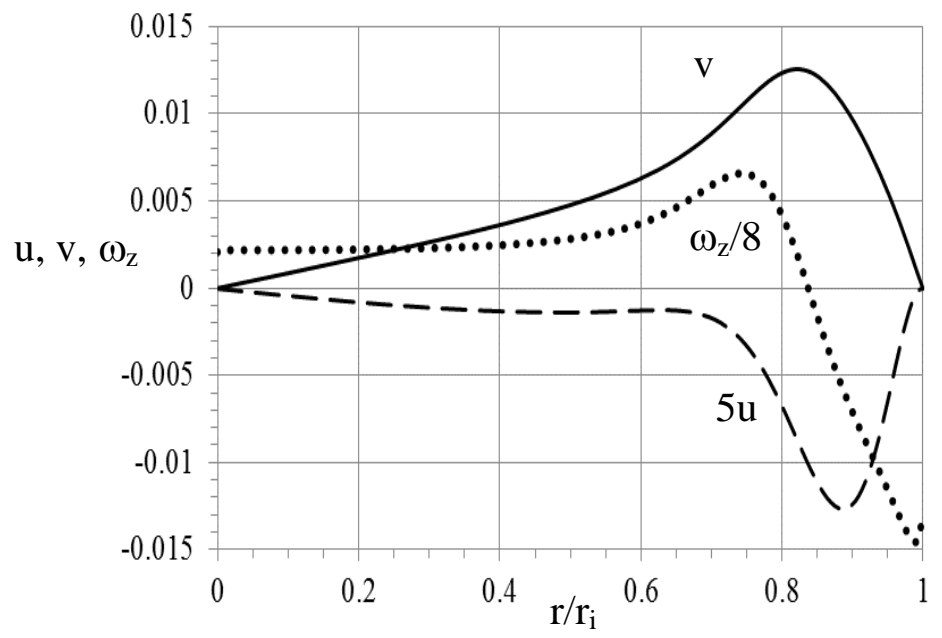

Fig. 11. Distribution on the interface of swirl $v$ and radial $u$ velocities, and the axial vorticity $\omega_{\mathrm{z}}$ at $R e=18000$ and $H_{\mathrm{w}}=0.8$. 


\section{Concluding remarks}

The goal of this paper is to explore the eddy development in a slow air-water flow with no interaction of these eddies with corner vortices. To this end, we chose a semispherical container, where the flow is driven by a rotating lid. It is found that as the water height $H_{\mathrm{w}}$ increases, the clockwise circulation emerges in water near the axis-bottom intersection at $H_{\mathrm{w}}=H_{\mathrm{ww}}=0.666$ (Fig. 2) and then the anticlockwise circulation emerges in air near the axis-interface intersection at $H_{\mathrm{w}}=H_{\mathrm{wa}}=0.732$ (Fig. 5). The new air region is a thin layer adjacent to the interface. Next the water clockwise circulation occupies the entire water domain at $H_{\mathrm{w}}=H_{\mathrm{w} 3}=0.898$ (Fig. 8). The air anticlockwise circulation expands up to the disk at $H_{\mathrm{w}}=H_{\mathrm{w} 4}=0.935$.

The topology of flow observed for $R e<<1$ remains invariant for moderate Re (Fig. 9 and Table 1). For large $R e$, the air anticlockwise circulation disappears and the bulk regions of the clockwise meridional circulation in air and water are separated by a thin layer of the water anticlockwise circulation.

Our study confirms that eddies can develops in creeping flows via the swirl decay mechanism and the cyclostrophic balance. The topological bifurcations we observe here are simpler and fewer than those found in cylindrical [9] and conical [10] containers where interaction of the kinematical and Moffatt eddies occurs.

In the spherical container only corner bubble-creation and corner-crossing topological bifurcations are found. Consequently, the flow topology in a spherical container is much more robust with respect to changes of control parameters. This might be an advantage in an industrial application as a bioreactor. 


\section{Acknowledgement}

Partial support from the Ministry of Science and Education and Junta de Andalucía (Spain) through grants nos. DPI2010-21103 and P08-TEP-04128, respectively, is gratefully acknowledged. Adnan Balci is supported by the Turkish Military Academy. 


\section{Appendix}

$\mathrm{u}$

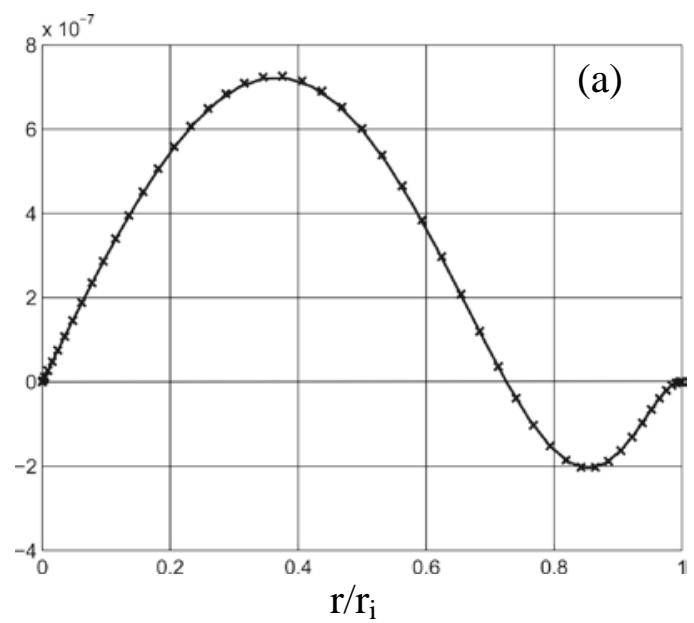

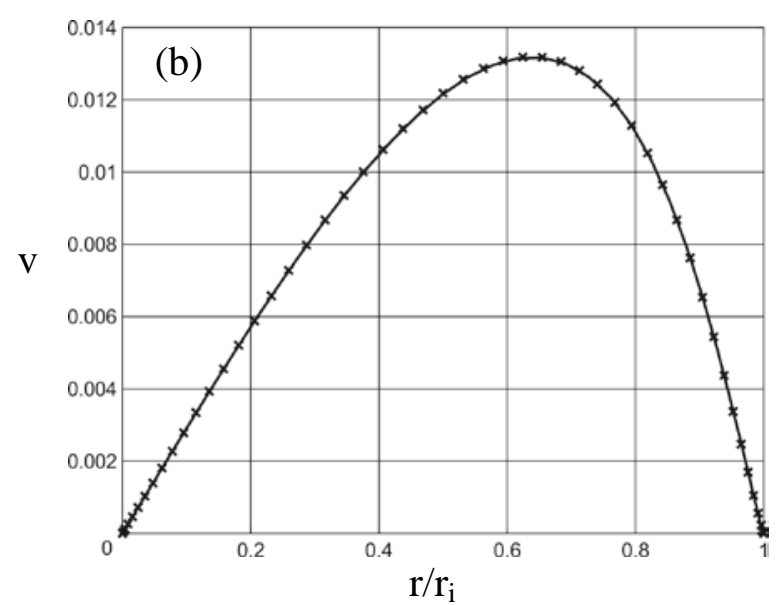

Fig. 12. Profiles of (a) radial and (b) swirl velocities on the interface at $R e=0.1$ and $H_{\mathrm{w}}=0.8$, obtained by using the standard (solid curves) and fine (crosses) grids.

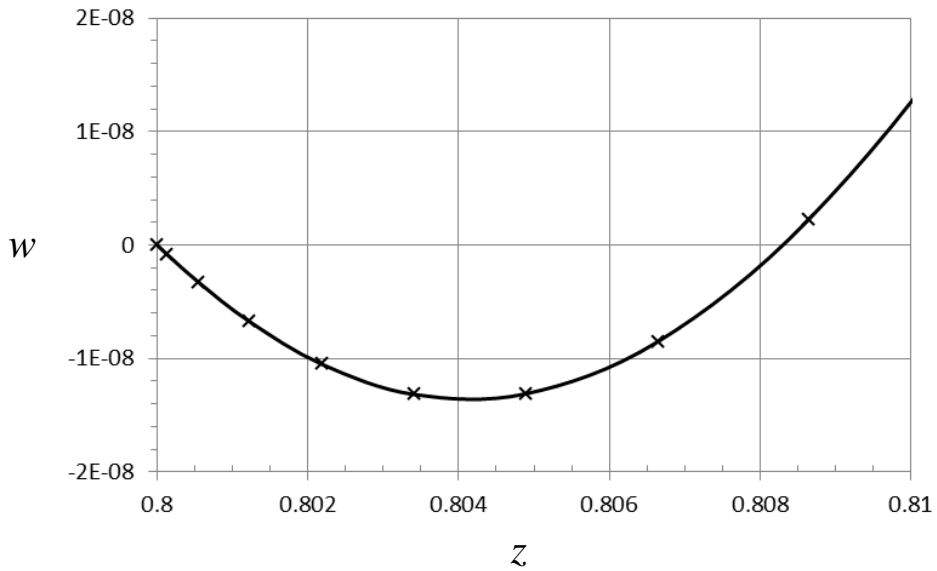

Figure 13. Distribution of grid points at z-axis within region $\mathrm{CR}_{\mathrm{a} 2}$ in Fig. 9(a) where $w<0$.

Figure 12 verifies that the obtained results are grid-independent. The profiles of (a) radial, u, and (b) swirl, v, velocities at $R e=0.1$ and $H_{\mathrm{w}}=0.8$ are plotted. The solid corves present the numerical results obtained with the standard grid, $\mathrm{n}_{\zeta \mathrm{w}}=40, \mathrm{n}_{\xi \mathrm{a}}=30$, and $\mathrm{n}_{\zeta}=50$, while the cross symbols present the numerical results obtained with the fine grid, $\mathrm{n}_{\zeta \mathrm{w}}=50, \mathrm{n}_{\xi \mathrm{a}}=40$, and $\mathrm{n}_{\zeta}=60$. 
The crosses merge with the curves within the accuracy of the drawing in Fig. 12 even for the radial velocity which is very small compared to the swirl velocity.

Figure 13 depicts the dependence of velocity at the axis in the z-range corresponding to region $\mathrm{CR}_{\mathrm{a} 2}$ in Fig. 9(a) where the axial velocity $w$ is negative. The cross symbols in Fig. 13 show the distribution of grid points whose number is sufficient for a satisfactory resolution of $\mathrm{CR}_{\mathrm{a} 2}$. 
[1] Moffatt, H. K. Viscous and resistive eddies near a sharp corner. J. Fluid Mech. 1964, 18, 1-18.

[2] Shankar, P. N. \& Deshpande, M. D. Fluid mechanics in the driven cavity. Annu. Rev. Fluid Mech. 2000 32, 93-136.

[3] Wakiya, S. Axisymmetric flow of a viscous fluid near the vertex of a body. J. Fluid Mech. 1976, 78, 737-747.

[4] Blake, J. On the generation of viscous toroidal eddies in a cylinder. J. Fluid Mech. 1979, 95, 209-222.

[5] Hills, C. P. Eddies induced in cylindrical containers by a rotating end wall. Phys. Fluids 2001, 13, 2279-2286.

[6] Hall, O., Hills, C. P. \& Gilbert, A. D. Slow flow between concentric cones. Quarterly J. of Mechanics \& App. Maths. 2007, 60, 27-48.

[7] Shtern, V. A flow in the depth of infinite annular cylindrical cavity. J. Fluid Mech. 2012, 711, 667-680.

[8] Shtern, V. N. Asymptotic flow in the depth of a narrow cavity. Phys. Fluids 2013, 25, 083604.

[9] Herrada, M. A. \& Shtern, V. N., 2014 Patterns of a creeping water-spout flow. J. Fluid Mech. 744, 65-88.

[10] Balci, A., Brøns, M., Herrada, M. A. \& Shtern, V. N., Vortex breakdown in a truncated conical bioreactor. Fluid Dyn. Res. 2015, 47(6) 065503.

[11] Ramazanov, Yu. A., Kislykh, V.I., Kosyuk, I.P., Bakuleva, N.V. \& Shchurikhina, V.V. 2007 Industrial production of vaccines using embryonic cells in gas-vortex gradient-less bioreactors. In book “New Aspects of Biotechnology and Medicine”, edited by A. M. Egorov, ISBN: 1-60021-465-7, pp. 87-91 (2007).

[12] Liow, K. Y. S., Tan, B. T., Thouas, G. \& Thompson, M. C. 2009 CFD modeling of the steady-state momentum and oxygen transport in a bioreactor that is driven by a rotating disk. Modern Physics Letters B 23 (2), 121-127.

[13] Lo Jacono, D., Nazarinia, M. and Brøns, M. Experimental vortex breakdown topology in a 
cylinder with a free surface. Phys. Fluids 2009, 21, 111704.

[14] Brøns, M., Voigt, L. K. \& Sorensen, J. N. 2001 Topology of vortex breakdown bubbles in a cylinder with a rotating bottom and a free surface. J. Fluid Mech. 428, 133-148.

[15] Brøns, M. 2007 Streamline topology: Patterns in fluid flows and their bifurcations. Advances in Applied Mechanics, 41,1-42.

[16] Bödewadt, U. T. 1940 Die Drehströmung über festem Grund”, Z. Angew. Math. Mech. 20, 241.

[17] Herrada, M. A., Shtern, V. N., \& López-Herrera, J. M. 2013 Vortex breakdown in a water-spout flow. Phys. Fluids 25, 093604. 